\title{
TNFR1-mediated signaling is important to induce the improvement of liver fibrosis by bone marrow cell infusion
}

\author{
Takuro Hisanaga • Shuji Terai - Takuya Iwamoto • \\ Taro Takami • Naoki Yamamoto • Tomoaki Murata • \\ Toshifumi Matsuyama • Hiroshi Nishina • Isao Sakaida
}

Received: 25 April 2011 /Accepted: 30 August 2011 /Published online: 11 October 2011

(C) The Author(s) 2011. This article is published with open access at Springerlink.com

\begin{abstract}
The importance of TNF- $\alpha$ signals mediated by tumor necrosis factor receptor type 1 (TNFR1) in inflammation and fibrosis induced by carbon tetrachloride $\left(\mathrm{CCl}_{4}\right)$, and in post-injury liver regeneration including a $\mathrm{GFP} / \mathrm{CCl}_{4}$ model developed as a liver repair model by bone marrow cell (BMC) infusion, was investigated. In mice in which TNFR1 was suppressed by antagonist administration or by knockout, liver fibrosis induced by $\mathrm{CCl}_{4}$ was significantly decreased. In these mice, intrahepatic macrophage infiltration and TGF- $\beta 1$ expression were reduced and stellate cell activity was decreased; however, expression of MMP-9 was also decreased. With GFP-positive BMC (TNFR1 wildtype, WT) infusion in these mice, fibrosis proliferation,
\end{abstract}

T. Hisanaga $\cdot$ S. Terai $(\bowtie) \cdot$ T. Iwamoto $\cdot$ T. Takami $\cdot$

N. Yamamoto $\cdot$ I. Sakaida

Department of Gastroenterology \& Hepatology,

Yamaguchi University Graduate School of Medicine,

Minami Kogushi 1-1-1,

Ube, Yamaguchi 755-8505, Japan

e-mail: terais@yamaguchi-u.ac.jp

T. Murata

Institute of Laboratory Animals, Yamaguchi University,

Minami Kogushi 1-1-1,

Ube, Yamaguchi 755-8505, Japan

T. Matsuyama

Department of Molecular Microbiology and Immunology,

Nagasaki University Graduate School of Biomedical Sciences,

1-12-4 Sakamoto,

Nagasaki 852-8523, Japan

H. Nishina

Department of Developmental and Regenerative Biology,

Medical Research Institute, Tokyo Medical and Dental University,

Yushima 1-5-45,

Bunkyo-ku, Tokyo 113-0034, Japan including host endogenous intrahepatic macrophage infiltration, TGF- $\beta 1$ expression and stellate cell activity, increased significantly. There was no significant increase of MMP-9 expression. In this study, TNFR1 in hosts had a promoting effect on $\mathrm{CCl}_{4}$-induced hepatotoxicity and fibrosis, whereas BMC infusion in TNFR1 knockout mice enhanced host-derived intrahepatic inflammation and fibrosis proliferation. These findings differed from those in WT recipient mice, in which improvement in inflammation and fibrosis with BMC infusion had previously been reported. TNFR1-mediated signaling might be important to induce the improvement of liver fibrosis by bone marrow cell infusion.

Keywords Tumor necrosis factor $\cdot \mathrm{CCl}_{4} \cdot$ Bone marrow cell $\cdot$ Liver cirrhosis $\cdot$ Hepatic stellate cell

\section{Introduction}

The mechanism of hepatotoxicity induced by many factors, including $\mathrm{CCl}_{4}$, has been studied for a long time (Drill 1952) and besides direct cytotoxicity due to these factors, enhanced hepatotoxicity due to inflammatory cell infiltration and stellate cell activation induced by these factors also occurs. TNF- $\alpha$ is an important inflammatory cytokine that induces hepatotoxicity; however, it also initiates liver regeneration after injury. This has been examined in various previous hepatotoxicity, hepatic resection and hepatic failure models. In addition, in recent stem cell studies, TNF- $\alpha$ signals have been found to be essential in the differentiation/proliferation process from stem cells to functional cells. For effective expression of TNF- $\alpha$ signals, of the various receptors, TNFR1 (p55) is important. In 
studies using TNFR1 knockout (KO) mice, while inhibition of hepatotoxicity has been shown, decreased liver regeneration and hepatocyte proliferation have also been confirmed (Gardner et al. 2003; Simeonova et al. 2001; Sudo et al. 2005; Yamada and Fausto 1998).

In our laboratory, using a $\mathrm{GFP} / \mathrm{CCl}_{4}$ model, $\mathrm{BMC}$ infusion has been shown to improve fibrosis and liver function in cirrhosis (Sakaida et al. 2004, 2008; Terai et al. 2002, 2005). In the liver of mice not treated with $\mathrm{CCl}_{4}$, without hepatic toxicity, repopulation of GFP-positive BMCs into the liver has not been observed, thus highlighting the need for chronic inflammatory signals in cell repopulation and effective expression. In addition, after BMC infusion, alterations in TNFR1 expression and associated increases in various cytokines such as fibroblast growth factor 2 (FGF2) have been recognized (Omori et al. 2004). This also suggested the importance of inflammatory signals, particularly TNF- $\alpha$ signals, in liver function improvement. Therefore, to investigate the importance of TNF- $\alpha$ signals mediated by TNFR1 in liver repair by BMC infusion in recipients, this study using TNFR1 antagonist-administered mice and TNFR1 KO mice as recipients was conducted.

\section{Materials and methods}

\section{Mice}

C57BL/6 TNFR1 KO mice were kindly provided by Hiroshi Nishina (Department of Developmental and Regenerative Biology, Medical Research Institute, Tokyo Medical and Dental University). TNFR1 KO mouse typing was performed by PCR analysis using primer typing box [p55R636 and HSV-TK (to confirm induction of silencing gene factor)]. Those primers were p55R636 (5'-GGCTGCAGTCCACGCACTGG-3'), and HSV-TK (5'-ATTCGCCAATGACAAGACGCTGG-3') (data not shown) (Pfeffer et al. 1993). C57BL/6 female mice were purchased from Chiyoda SLC (Tokyo, Japan). GFP-transgenic mice (TgN( $\beta$-act-EGFP)Osb) were kindly provided by Masaru Okabe (Genome Research Center, Osaka University, Osaka, Japan). These mice were maintained in specific pathogen-free housing at the Animal Experiment Facility of Yamaguchi University School of Medicine and cared for in accordance with the animal ethics requirements at Yamaguchi University School of Medicine.

\section{Experimental protocol}

First, to confirm that signals mediated by TNFR1 are important in intrahepatic inflammatory responses and fibrosis induced by $\mathrm{CCl}_{4}$, an experiment using TNFR1 antagonist (anti-mouse TNFR1 antibody, MAB430; R\&D Systems, Minneapolis, MN, USA) was conducted in wildtype (WT) mice. In WT mice, $\mathrm{CCl}_{4}$ was administered for 4 weeks to create a liver cirrhosis model; then, by caudal vein injection of $100 \mu \mathrm{g} /$ body of antagonist, signals mediated by TNFR1 were suppressed in recipients; and thereafter, $\mathrm{CCl}_{4}$ was continued for 1 week. A model was also created with infusion of GFP-positive BMCs after $1 \mathrm{~h}$, when in vivo activity of the antagonist reaches a peak. There were 3 groups: WT (Control) (wild-type, without BMC infusion); WT + A (wild-type, with TNFR1 antagonist only, without BMC infusion); and GFP/WT+A (wildtype, with TNFR1 antagonist and with BMC infusion) (Fig. 1).

As will be described later, by blocking TNFR1, suppression of fibrosis and suppression of inflammatory cell infiltration were confirmed. Therefore, as a more highly specific model, a model was created by the following protocol with TNFR1 KO mice as BMC infusion recipients.

Six-week-old female C57BL/6 mice and female isogenic TNFR1 KO mice were treated with $\mathrm{CCl}_{4}(1.0 \mathrm{ml} / \mathrm{kg}$ body diluted 1:3 in corn oil) twice a week for 8 weeks. In the other group, after 4 weeks of $\mathrm{CCl}_{4}$ administration in each group (C57BL/6 wild-type and TNFR1 KO), bone marrow cells $(\mathrm{BMC})\left(1 \times 10^{5}\right.$ cells $)$ from GFP transgenic mice were injected via the tail vein as previously described (Terai et al. 2003).

After 8 weeks, $36 \mathrm{~h}$ after the last $\mathrm{CCl}_{4}$ injection, the mice were sacrificed to examine the blood data and liver tissue specimens. The liver was fixed in $4 \%$ buffered paraformaldehyde for 24-48 $\mathrm{h}$ and paraffin embedded. Blood samples were obtained by cardiac puncture and drawn into a glass tube containing 7.5\% EDTA ( $\mathrm{pH}$ 7.4). After centrifugal separation, the plasma was stored at $4^{\circ} \mathrm{C}$.

There was a total of 4 groups in this study: WT (Control), wild-type without BMC infusion; $\mathrm{KO}$ (Control), TNFR1 KO without BMC infusion; GFP/WT, wild-type with GFP-positive BMC infusion; and GFP/KO, TNFR1 $\mathrm{KO}$ with GFP-positive BMC infusion (Fig. 1).

Quantitative analysis of liver fibrosis and immunohistochemistry

The liver fibrosis area was quantified with Sirius-red staining using an Olympus Provis microscope equipped with a CCD camera (Olympus, Tokyo, Japan). The red area, considered the fibrotic area, was assessed by computer-assisted image analysis with MetaMorph software (Universal Imaging, Downingtown, PA, USA) at a magnification of $\times 40$. The mean value of 10 randomly selected areas per sample was used as the expressed percent area of fibrosis. 


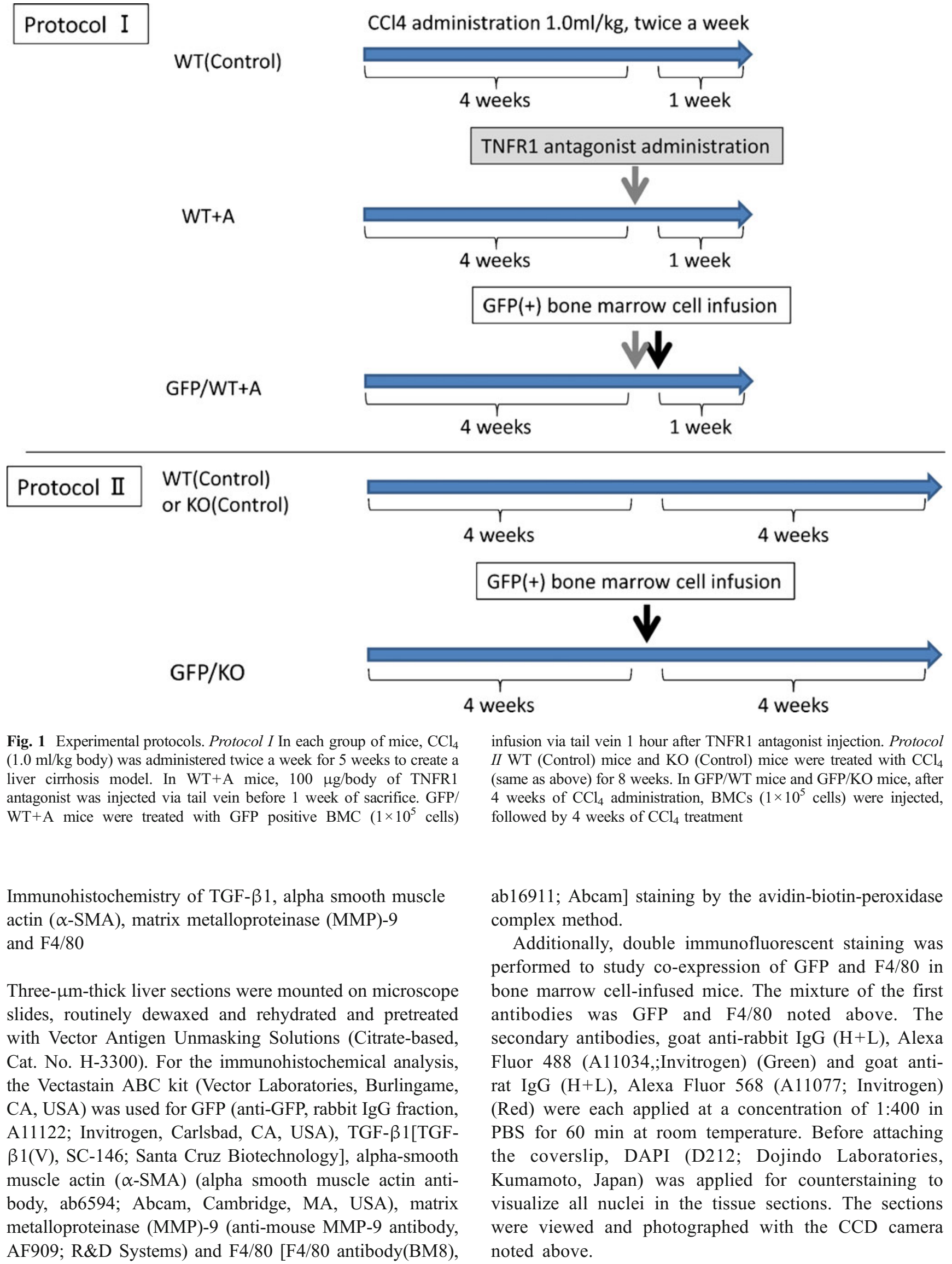


Real-time quantitative PCR analysis

Total RNA was isolated from the livers of the mice treated at 4 weeks after the BMC infusion or control $\mathrm{CCl}_{4}$ treatment. The messenger RNA (mRNA) expressions of TGF- $\beta 1$ and MMP-9 were evaluated using real-time quantitative PCR. Total RNA was extracted using the RNeasy Mini Kit (Qiagen, Hilden, Germany). For cDNA synthesis, AMV reverse transcription reagents were used according to the manufacturer's instructions (Roche Diagnostic, Pleasanton, CA, USA). Real-time PCR was performed with SYBR Green Master Mix (Roche Diagnostic). The primers used for TGF- $\beta 1$ were $5^{\prime}$-GAAGCCATCCGTGGCCAGAT-3' (forward) and 5'-GACGTCAAAAGACAGCACT-3' (reverse), for MMP-9 were 5'-GGAACTCACACGACATCTTCCA-3' (forward) and 5'-GAAACTCACACGCCAGAAGAATTT-3' (reverse) and collagen type 1 alpha were $5^{\prime}$-CGGGCAG GACTTGGGTA-3' (forward) and 5'-CGGAATCT GAATGGTCTGACT-3' (reverse). The PCR primers used for mouse glyceraldehyde-3-phospatase dehydrogenase (GAPDH), which was used as an internal control, were: 5'GTCTTCACCACCATGGAGAAGGC-3', 5'-ATG CCAGTGAGCTTCCCGTTCAGC-3'. The cycle for PCR was as follows: 1 cycle of $95^{\circ} \mathrm{C}$ for $20 \mathrm{~s} ; 40$ cycles of $3 \mathrm{~s}$ at $95^{\circ} \mathrm{C}$ and $30 \mathrm{~s}$ at $60^{\circ} \mathrm{C}$; and 1 cycle of $95^{\circ} \mathrm{C}$ for $15 \mathrm{~s}, 60^{\circ} \mathrm{C}$ for $1 \mathrm{~min}$, and $95^{\circ} \mathrm{C}$ for $15 \mathrm{~s}$. Reactions were performed in a Step One Plus ${ }^{\mathrm{TM}}$ real-time PCR system (Applied Biosystems, California, CA, USA) and amounts of all mRNAs were quantified using StepOne ${ }^{\mathrm{TM}}$ version 2.1 software (Applied Biosystems).

\section{Western blot analysis}

The liver samples (approximately $40 \mathrm{mg}$ ) were homogenized in $1 \mathrm{ml}$ of cell lysis buffer (Cell Signal Technology, Beverly, MA, USA) and $1 \mathrm{mM}$ phenylmethanesulfonyl fluoride (PMSF) and complete mini-centrifuged (Roche Diagnostic). The supernatant represented the whole protein. Then, $40 \mu \mathrm{g}$ of the protein sample were mixed with the same volume of loading buffer (5\% 2-mercaptoethanol and 95\% Laemmli Sample Buffer; Bio-Rad Laboratories, Hercules, CA, USA), heated for $4 \mathrm{~min}$ at $97^{\circ} \mathrm{C}$ and separated by $10 \%$ SDS-PAGE. The separated bands were transferred to Immobilon-P transfer membrane (Millipore, Billerica, MA, USA), followed by blocking of the membranes for $1 \mathrm{~h}$ with blocking buffer $(0.1 \%$ Tween-20; Wako Pure Chemical Industries, Osaka, Japan), 0.2\% IBlock $^{\mathrm{TM}}$ reagent (Tropix, Bedford, MA, USA) and $1 \mathrm{mM}$ Tris- $\mathrm{HCl}$ buffer ( $\mathrm{pH}$ 7.5) (Invitrogen). The membranes were then washed with washing buffer $(0.1 \%$ Tween-20, $1 \mathrm{mM}$ Tris- $\mathrm{HCl}$ buffer; $\mathrm{pH}$ 7.5) and incubated overnight at $4{ }^{\circ} \mathrm{C}$ with the primary antibodies against MMP-9 (R\&D Sytems), TGF- $\beta 1$ (Santa Cruz Biotechnology) and $\beta$-actin (Abcam) in blocking buffer. Then, after being washed, the membranes were incubated for $1 \mathrm{~h}$ at room temperature with the appropriate secondary antibodies. Reactive bands were identified using ECL (Amersham Biosciences, Piscataway, NJ, USA) and autoradiography, according to the manufacturer's instructions.

\section{Statistical analysis}

Statistical significance was determined using 2-tailed Student's $t$ test. Results are presented as the mean \pm standard deviation and differences of $p<0.05$ were considered significant.

\section{Results}

Alterations of liver fibrosis and intrahepatic inflammatory invasion by TNFR1 antagonist and BMC infusion

At 1 week after TNFR1 antagonist administration in wildtype mice, liver fibrosis was significantly suppressed [WT (Control): $\mathrm{WT}+\mathrm{A}, p<0.01]$ in the antagonist-treated group compared to the untreated group. In addition, F4/80positive intrahepatic macrophage infiltration tended to be suppressed [WT (Control): WT $+\mathrm{A}, p=0.07$ ] and stellate cell activity, shown by $\alpha$-SMA staining, was significantly suppressed [WT (Control): WT $+\mathrm{A}, p<0.01]$. However, in the group with GFP-positive BMC infusion in addition to the antagonist, liver fibrosis did not improve with BMC infusion (WT+A: GFP/WT $+\mathrm{A}, p=0.44)$ Conversely, both macrophage infiltration (WT+A: $\mathrm{GFP} / \mathrm{WT}+\mathrm{A}, p=0.02$ ) and stellate cell activity (WT $+\mathrm{A}: \mathrm{GFP} / \mathrm{WT}+\mathrm{A}, p<0.01)$ increased significantly (Fig. 2a-1).

Liver fibrosis did not improve in the $\mathrm{KO}$ recipient model

The migration of GFP positive cells was confirmed using immunohistochemistry of GFP (Fig. 3a, b). On Sirius red staining, liver fibrosis was significantly suppressed in KO (Control) mice compared to WT (Control) mice [WT (Control): KO (Control), $p<0.05]$. WT mice tended to have strong fibrosis but similar to the $\mathrm{GFP} / \mathrm{CCl}_{4}$ model that we previously reported (Terai, et al. 2003), with BMC infusion, fibrosis improved significantly [WT (Control): GFP/WT, $p<0.01]$. However, in TNFR1 KO mice, in whom fibrosis was suppressed, BMC infusion did not improve liver fibrosis [KO (Control): GFP/KO, $p=$ 0.91] (Fig. 3c-g).

In addition, on real-time RT-PCR for collagen I- $\alpha 1$, in WT mice, fibrosis proliferation was increased and in $\mathrm{KO}$ mice, it was suppressed [WT (Control): KO (Control), $p<$ 0.05]. In WT mice, as in our previous $\mathrm{GFP} / \mathrm{CCl}_{4}$ model, 

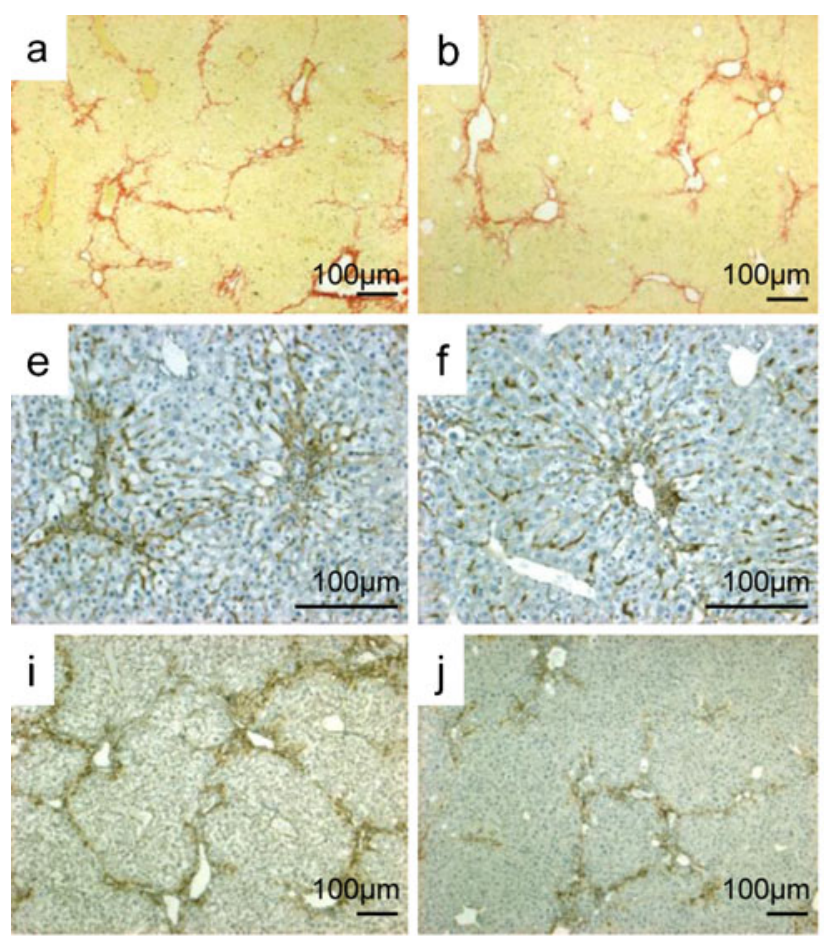

Fig. 2 Suppression of liver fibrosis, hepatic stellate cell activation and F4/80 positive cell invasion using TNFR1 antagonist. a-c Sirius red staining of liver sections (original magnifications $\times 40$ ). d Statistical analysis of fibrotic area by the staining. Scale bars standard deviations. WT liver (a) had severe fibrosis but WT + A liver (b) and GFP/WT+A liver (c) did not. There was no significant improvement induced by BMC infusion with TNFR1 antagonist. e-g Immunohistochemistry of F4/80 (original magnifications $\times 100$ ). h Statistical

after BMC infusion, collagen I- $\alpha 1$ mRNA expression was decreased [WT (Control): GFP/WT, $p<0.05$ ]. On the other hand, there were no changes in $\mathrm{KO}$ mice with $\mathrm{BMC}$ infusion [KO (Control): GFP/KO, $p=0.67]$ (Fig. 3h).

Fibrolytic reactions induced by BMC infusion

Expression of MMP-9, important in liver fibrosis improvement, corresponded with sites of inflammation and fibrosis in each model and based on western blot and real-time RTPCR analysis, MMP-9 expression was significantly suppressed in $\mathrm{KO}$ mice compared to wild-type mice [WT (Control): $\mathrm{KO}$ (Control), $p<0.01]$. However, in $\mathrm{KO}$ mice, even with GFP-positive BMC infusion, there was no significant increase, statistically proven in Real-time RTPCR analysis [KO (Control): GFP/KO, $p=0.41]$. This
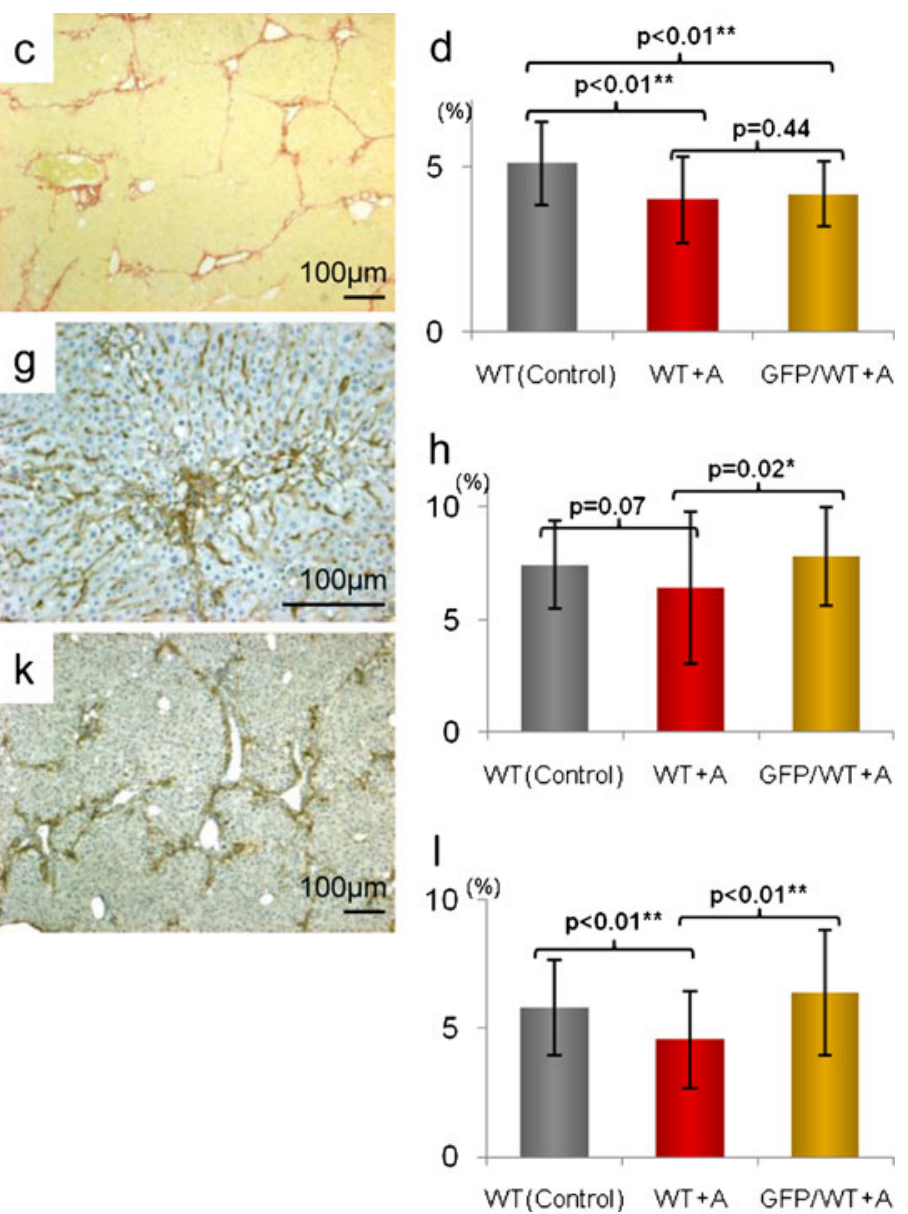

analysis of F4/80 positive area. Comparing WT (e) with WT+A (f), TNFR1 antagonist suppressed the invasion of F4/80 positive cells into the liver but in GFP/WT $+\mathrm{A}(\mathbf{g})$, the invasion increased. $\mathbf{i}-\mathbf{k}$ Immunohistochemistry of $\alpha$-SMA (original magnifications $\times 40$ ). I Statistical analysis of $\alpha$-SMA positive area. Comparing WT (i) with $\mathrm{WT}+\mathrm{A}(\mathbf{j})$, TNFR1 antagonist suppressed the expression of $\alpha$-SMA but in GFP/WT+A (k), the bone marrow cell infusion raised the activation regardless of TNFR1 antagonist administration

showed that, in KO mice, although MMP-9 expression is suppressed, BMC infusion in these mice did not clearly increase MMP-9 (Fig. 3i-n).

Alterations of F4/80 positive cells into TNFR1 KO mice

In KO (Control) mice, compared to WT (Control) mice, intrahepatic infiltration of F4/80-positive cells (macrophages) induced by $\mathrm{CCl}_{4}$ was significantly suppressed [WT (Control): KO (Control), $p<0.01]$ but with BMC infusion, this infiltration was significantly increased $[\mathrm{KO}$ (Control): GFP/KO, $p<0.01$ ] (Fig. 4a-e). Upon evaluation of macrophage origin, in double immunofluorescent staining (GFP: emitting green light, and F4/80: emitting red light), no cells showed co-positivity with GFP and F4/80. It was indicated that the increased F4/80-positive macro- 

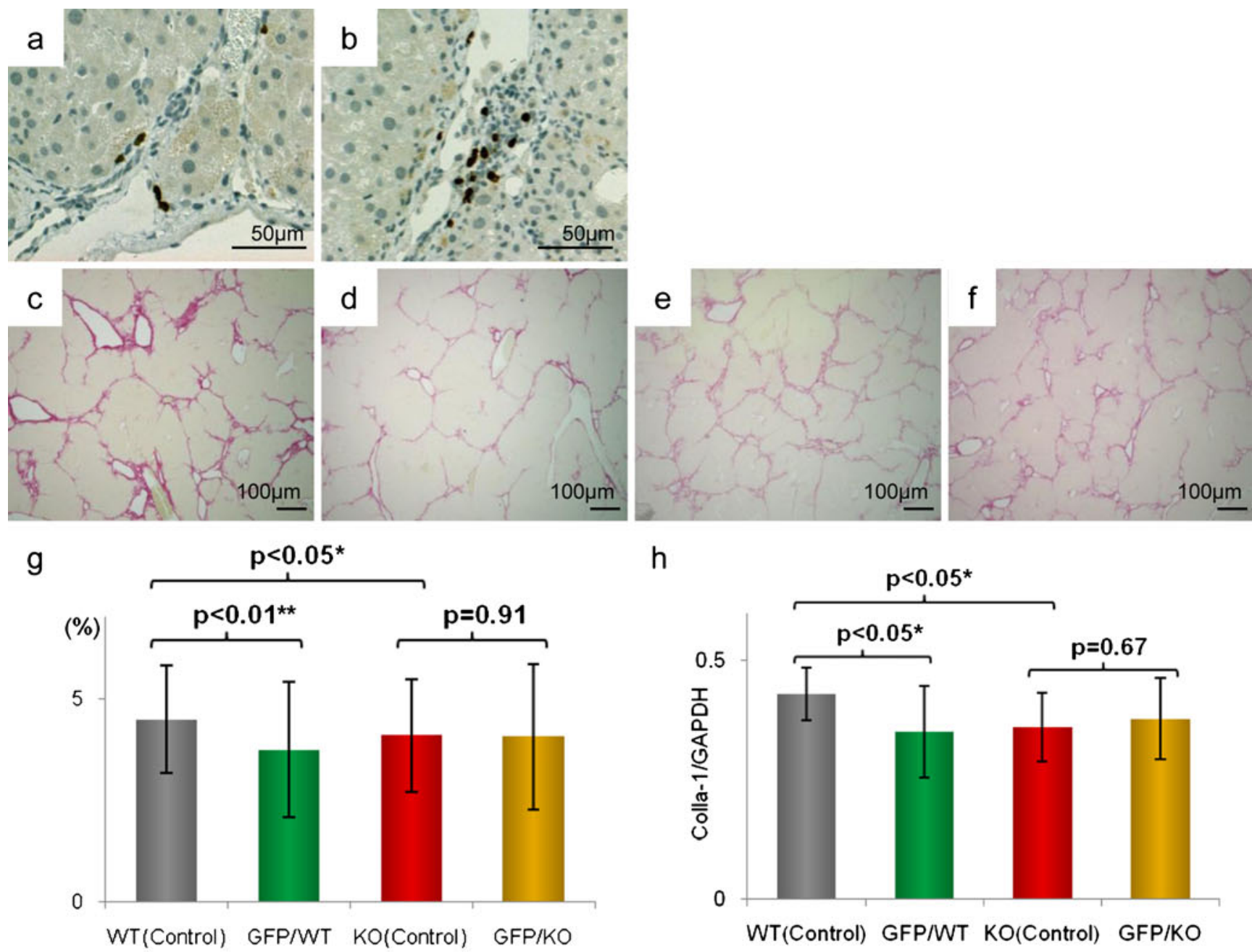

h
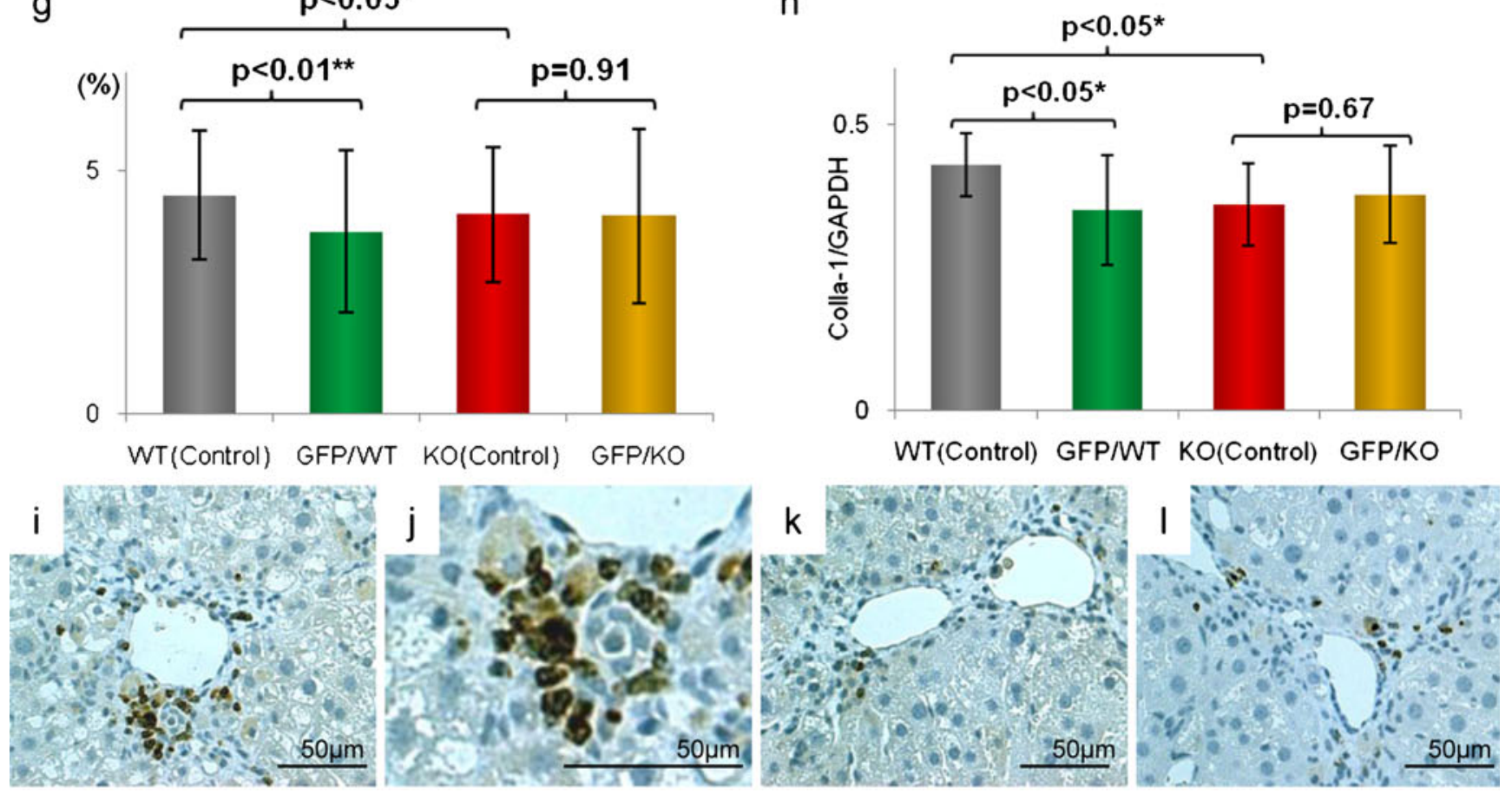

$\mathrm{m}$

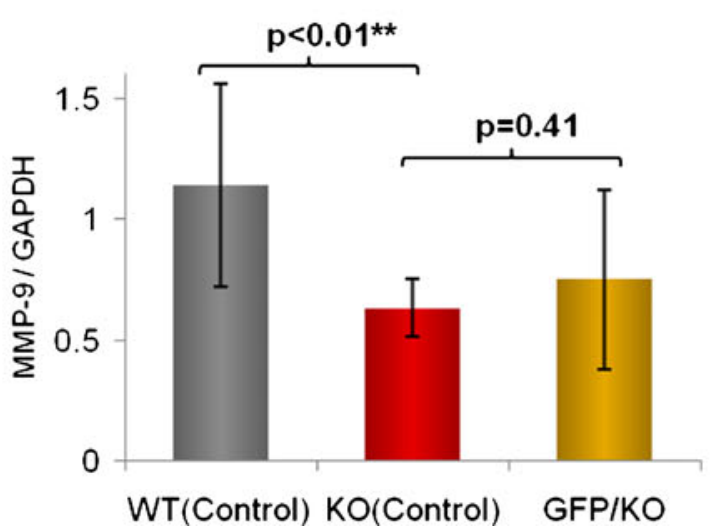

$\mathrm{n}$

MMP-9

$\beta$-actin

WT(Control) $\mathrm{KO}$ (Control) GFP/KO 
4 Fig. 3 Comparison of liver fibrosis using TNFR1 KO mice. a, b antiGFP immunohistochemistry (original magnifications, $\times 200$ ). Both in wild type mouse live (a) and in TNFR1 KO mouse liver (b), the migrations of GFP positive bone marrow cells were confirmed. $\mathbf{c}-\mathbf{f}$ Sirius red staining of liver sections (original magnifications, $\times 40$ ). $\mathbf{g}$ Statistical analysis of fibrotic area. Scale bars standard deviations. Compared with WT (Control) (c), GFP/WT (d) had marked fibrolysis. KO (Control) (e) had significantly suppressed fibrosis but there was no marked improvement of fibrosis in GFP/KO (f). $\mathbf{h}$ Real-time RTPCR analysis of expression of collagen type 1 alpha-1, indicating the fibrotic activity, showed similar results. i-I Immunohistochemistry of MMP-9 in liver sections (original magnifications, $\times 200$ ). Liver section of WT (Control) (i) [magnified figure $(\times 400)(\mathbf{j})$ ] had certain degree of expression but in KO (Control) liver (k) and in GFP/KO liver (l), the expression was significantly low level. $\mathbf{m}$ Real-time RT-PCR analyses. n Western blot analyses of MMP-9; both showed suppression in TNFR1 KO mice but no significant increase in GFP/KO mice was seen and the suppression remained

phages were derived from differentiation and proliferation of recipient pre-existing cells (Fig. 4f).

Upregulation of TGF- $\beta 1$ signals and hepatic stellate cell activation

Upon evaluation by immunostaining, TGF- $\beta 1$ expression was decreased in $\mathrm{KO}$ (Control) mice but after BMC infusion, it was significantly increased (Fig. 4g-j). On real-time RT-PCR analysis as well, in KO mice, TGF- $\beta 1$ increased after BMC infusion [KO (Control): GFP/KO, $p<$ 0.05] (Fig. 4k). On western blot analysis, the findings were similar (Fig. 41). With alterations in TGF- $\beta 1$ expression, stellate cell activity, evaluated by $\alpha$-SMA staining, was suppressed in $\mathrm{KO}$ (Control) mice but with BMC infusion, in $\mathrm{GFP} / \mathrm{KO}$ mice, it was significantly increased $[\mathrm{KO}$ (Control): GFP/KO, $p<0.05$ ] (Fig. $4 \mathrm{~m}-\mathrm{p}$ ).

\section{Discussion}

TNF- $\alpha$ has long been known as an inflammatory cytokine whose action is mediated mainly by TNFR1. In addition to TNFR1, several other receptors exist, including TNFR2, each with a variety of actions, including cytotoxic effects, cell proliferation and protective effects. TNFR1 itself is known to be involved in various actions, including induction of hepatocyte apoptosis, extracellular matrix proliferation via downstream signals, anti-apoptosis effects and hepatocyte proliferation. In a previous partial hepatectomy model, the importance of TNF- $\alpha$ in liver regeneration was demonstrated (Yamada et al. 1997) but on the other hand, in studies using hepatotoxic factors such as $\mathrm{CCl}_{4}$ and LPS, the involvement of TNF- $\alpha$-TNFR1 pathways in the enhancement of liver cell injury and fibrosis has often been reported (Simeonova et al. 2001; Sudo et al. 2005; Horn et al. 2000). For applications of these findings to clinical liver disease, studies using TNFR1 knockout models, anti-TNF$\alpha$ neutralizing antibodies and anti-TNFR1 antagonists have been conducted. Reductions in liver injury and liver fibrosis have been reported (Haak-Frendscho et al. 1994; Inui et al. 2005; Shibata et al. 2008a).

In the $\mathrm{GFP} / \mathrm{CCl}_{4}$ model, we reported that BMC infusion has liver repair effects and improves liver fibrosis (Terai et al. 2003). In reports to date from our laboratory, we have shown that inflammatory cytokines induced by $\mathrm{CCl}_{4}$, primarily TNF- $\alpha$, are important in BMC survival and liver repair (Omori et al. 2004). In addition, at other facilities, in vitro models have shown the importance of TNF- $\alpha$ signals in the differentiation of bone marrow stem cells to functional cells. There are various theories about BMC kinetics when infused in vivo (Higashiyama et al. 2007; Dalakas et al. 2009; Terai and Sakaida 2008; Khurana and Mukhopadhyay 2007) but much remains unknown.

In our study, to first confirm the effects of TNFR1, a TNFR1 antagonist was used. Because TNFR1 antagonists differ from anti-TNF- $\alpha$ antibodies, only block TNFR 1 , and do not affect other signal pathways such as TNFR2 (Shibata et al. 2008b), administration of a sufficient dose of antagonist in wild-type mice can reproduce a TNFR1 KO mouse system. A dose of $100 \mu \mathrm{g} /$ body is sufficient to enable inhibition of intrahepatic inflammatory responses and liver fibrosis (Shibata et al. 2008a). Furthermore, the in vivo half-life of an intravenously administered TNFR1 antagonist is 5-6 days (Haak-Frendscho et al. 1994), so the blocking effect of TNFR1, up to the time of tissue sampling after 1 week, as used in the present protocol, probably continued.

In mice administered with the TNFR1 antagonist, intrahepatic macrophage infiltration was suppressed and stellate cell activation was decreased. Studies in liver injury suppression models, by inhibition of TNF $\alpha$-TNFR1 signals, not only with TNFR1 antagonists but also with anti-TNF- $\alpha$ antibodies, have been reported by several facilities (Sudo et al. 2005; Yamada and Fausto 1998; Shibata et al. 2008a; Kitamura et al. 2002; Bahcecioglu et al. 2008). In the present study as well, in a model of advanced liver fibrosis induced by $\mathrm{CCl}_{4}$ administration in wild-type mice, the TNFR1 antagonist suppressed intrahepatic inflammatory cell infiltration and improved liver fibrosis. However, in this model, with combined GFP-positive BMC infusion, intrahepatic inflammatory cell infiltration and stellate cell activation, which were suppressed by the antagonist, worsened with BMC infusion (attenuation of the suppression effect).

Next, to further clarify this phenomenon, TNFR1 KO mice were used as recipients to investigate whether there were differences in liver repair with BMC infusion based on the presence or absence of TNFR1. In GFP/KO mice, using TNFR1 KO mice as recipients, no liver repair effects with BMC infusion were observed. In TNFR1 KO mice, 

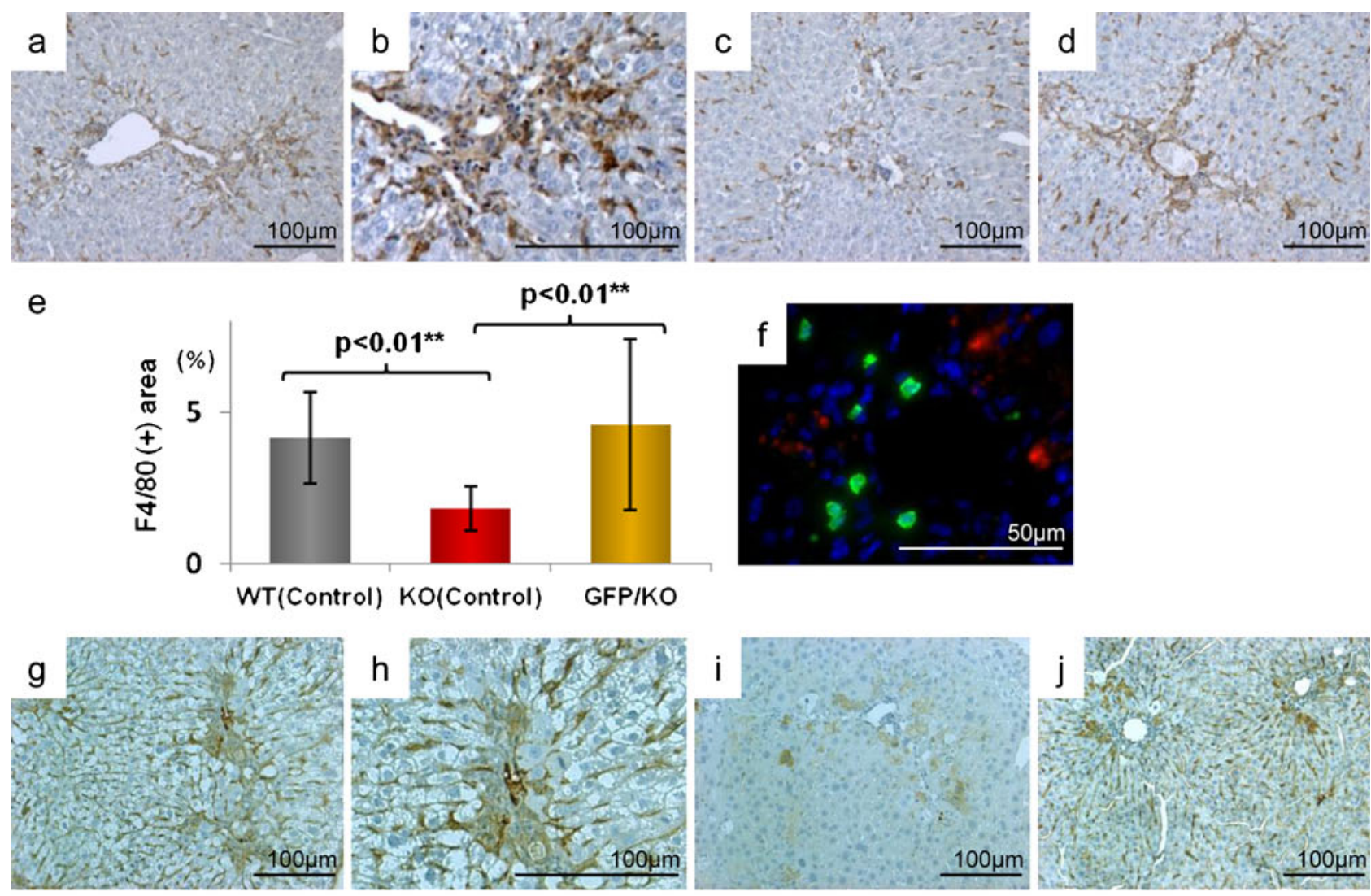

k

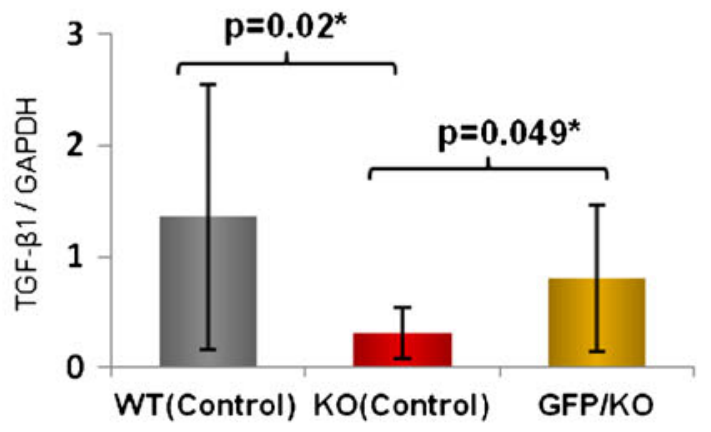

I

TGF- $\beta 1$

$\beta$-actin
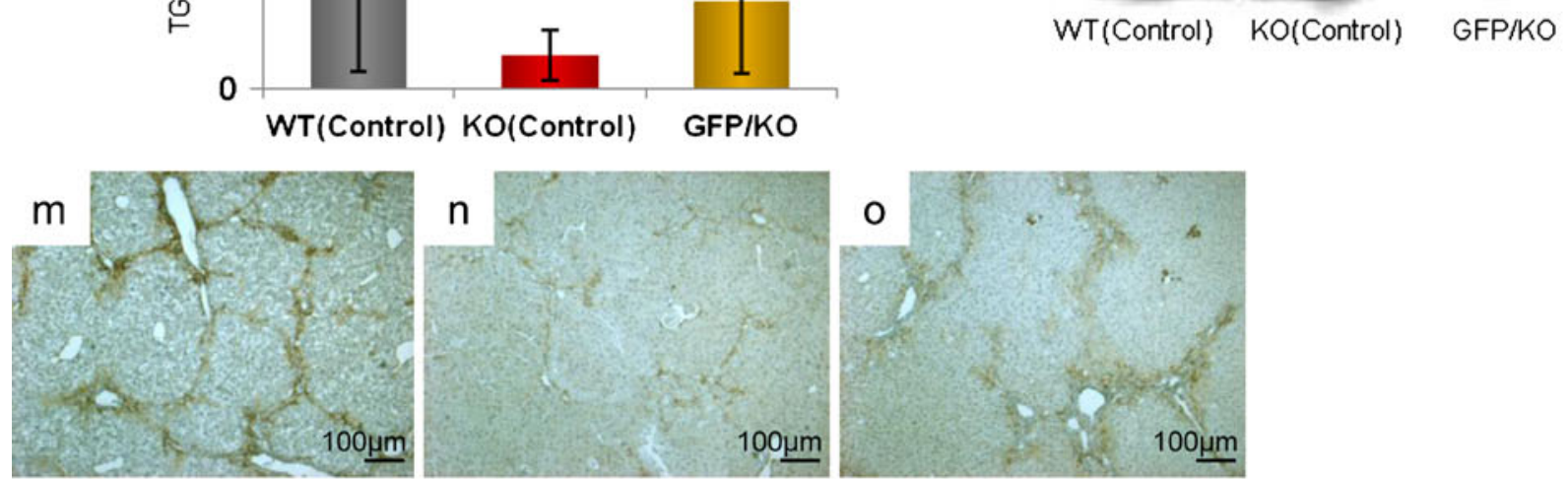

p

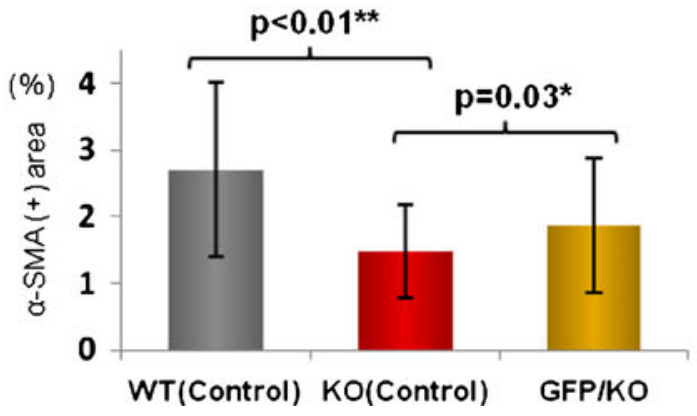


Fig. 4 Alteration of F4/80-positive cell infiltration, TGF- $\beta 1$ expression and hepatic stellate cell activation. a-d Immunohistochemical analysis of F4/80 (original magnifications, $\times 100$ ). e Statistical analysis of F4/80 positive area. Scale bars standard deviations. Compared with WT (Control) (a) [magnified figure $(\times 200)(\mathbf{b})$ ], the invasion of F4/80 positive cells was markedly suppressed in $\mathrm{KO}$ (Control) (c) and significantly increased in GFP/KO (d). f Double fluorescent immunohistochemistry of GFP and F4/80 (original magnifications, $\times 400$ ). GFP was marked with FITC (green) and F4/80 with Cy3 (red). This showed the mismatch between the GFP-positive area and that of F4/ 80. g-j Immunohistochemical analyses of expression (original magnifications, $\times 100)$. WT (Control) (g) [magnified figure $(\times 200)$ (h)] had much expression but in KO (Control) (i), it was suppressed. Adversely, GFP/KO (j) had more TGF- $\beta 1$ expression than $\mathrm{KO}$ (Control). k Real-time RT-PCR analyses of TGF- $\beta 1$. I Western blot analyses using TGF- $\beta 1$ antibody. Both showed the increase of TGF- $\beta 1$ expression in $\mathrm{GFP} / \mathrm{KO}$, suppressed in $\mathrm{KO}$ (Control). m-o Immunohistochemical analyses of $\alpha$-SMA (original magnifications $\times 40$ ). $\mathbf{p}$ Statistical analysis of $\alpha$-SMA positive area. Scale bars standard deviations. Compared with WT (Control) (m), KO (Control) (n) had less hepatic stellate cell activation but the activation was markedly increased in GFP/KO (o)

compared to wild-type mice, although $\mathrm{CCl}_{4}$-induced hepatotoxicity and liver fibrosis were suppressed, GFP-positive BMC infusion showed no synergism in liver repair or antihepatotoxic effects.

The importance of an increase in hepatic expression of MMP-9 by BMC infusion in liver fibrosis improvement has been reported by many facilities, including our laboratory (Sakaida et al. 2004, 2008; Higashiyama et al. 2007; Alwayn et al. 2008). In the present study, MMP-9 expression was markedly suppressed in TNFR1 KO mice compared to wildtype mice. MMP-9 expression has been shown to increase by TNF- $\alpha$ signals mediated via TNFR1 (Robinson et al. 2002; Vaday et al. 2001). In TNFR1 KO mice, the fact that this was suppressed is a consistent finding. In our laboratory, GFPpositive BMC infusion in wild-type mice was reported to enhance hepatic expression of MMP-9 (Sakaida et al. 2004). Within this series of cascades, inflammatory signals such as TNFR1 have been shown to be important (Omori et al. 2004). On the other hand, in GFP/KO mice, in which GFPpositive BMCs were infused in TNFR1 KO mice, there was no significant increase in MMP-9 expression and, moreover, the amount of expression compared to wild-type mice was significantly decreased, with no demonstrable effect on liver fibrolysis. With regard to the origin of MMP-9, infused BMCs have received attention but signals mediated by TNFR1 in pre-existing hepatocytes and host-derived hepatic macrophages are also important (Haruyama et al. 2000). These are suppressed in KO mice; furthermore, an increase in cytokine groups, such as increased TGF- $\beta 1$ expression, which conversely suppresses MMP-9 expression, also has an influence (Vaday et al. 2001).

In TNFR1 KO mice, macrophage infiltration, increased TGF- $\beta 1$ and enhanced $\alpha$-SMA activity were noted. But no cells showing co-positivity with GFP staining were con- firmed in F4/80 immunohistochemistry. We think the increase in macrophage came from recipient macrophage. In addition, as a result, other inflammatory signals such as TGF- $\beta 1$ increased, followed by enhanced stellate cell activity and increased liver fibrosis (Parsons et al. 2007; Moreira 2007). DNA-Chip analysis also showed that the macrophage-related gene such as "monocyte-macrophage differentiation, MMD" was activated in TNFR1 KO mice by bone marrow cell infusion (data not shown). Several reports have evaluated the relationship between infiltrating macrophages in the liver with hepatotoxicity, liver fibrosis and liver regeneration (Liu et al. 2010; Duffield et al. 2005; Tacke et al. 2009). An association between intrahepatic macrophages with liver fibrolysis has also been reported (Fallowfield et al. 2007). Based on the present study, after BMC infusion in TNFR1 KO recipients, macrophages infiltrating the liver enhanced the above-mentioned inflammatory responses and increased liver fibrosis. In conclusion, proper TNFR1 signaling might be a key factor to induce the improvement of liver fibrosis by bone marrow cell infusion. Further studies will be needed to understand the precise mechanism.

Acknowledgements Ms. Mariko Yamada, Ms. Isako Fujimoto and Ms. Yoko Fukuzumi helped us with the cell culture and immunohistochemical analysis. This study was supported by Grants-in-Aid for scientific research from the Japan Society for the Promotion of Science (No. 22390150) and the Ministry of Health, Labour and Welfare, the Knowledge Cluster Initiative and Japan Science and Technology agency.

Open Access This article is distributed under the terms of the Creative Commons Attribution Noncommercial License that permits any noncommercial use, distribution and reproduction in any medium, provided the original author(s) and source are credited.

\section{References}

Alwayn I, Verbesey J, Kim S et al (2008) A critical role for matrix metalloproteinases in liver regeneration. J Surg Res 145:192-198

Bahcecioglu I, Koca S, Poyrazoglu O et al (2008) Hepatoprotective effect of infliximab, an anti-TNF-alpha agent, on carbon tetrachloride-induced hepatic fibrosis. Inflammation 31:215-221

Dalakas E, Newsome P, Boyle S, et al. (2009) Bone marrow stem cells contribute to alcohol liver fibrosis in humans. Stem Cells Dev

Drill V (1952) Hepatotoxic agents; mechanism of action and dietary interrelationship. Pharmacol Rev 4:1-42

Duffield J, Forbes S, Constandinou C et al (2005) Selective depletion of macrophages reveals distinct, opposing roles during liver injury and repair. J Clin Invest 115:56-65

Fallowfield JA, Mizuno M, Kendall TJ et al (2007) Scar-associated macrophages are a major source of hepatic matrix metalloproteinase-13 and facilitate the resolution of murine hepatic fibrosis. J Immunol 178:5288-5295

Gardner C, Laskin J, Dambach D et al (2003) Exaggerated hepatotoxicity of acetaminophen in mice lacking tumor necrosis factor receptor-1. Potential role of inflammatory mediators. Toxicol Appl Pharmacol 192:119-130 
Haak-Frendscho M, Marsters S, Mordenti J et al (1994) Inhibition of TNF by a TNF receptor immunoadhesin. Comparison to an antiTNF monoclonal antibody. J Immunol 152:1347-1353

Haruyama T, Ajioka I, Akaike T et al (2000) Regulation and significance of hepatocyte-derived matrix metalloproteinases in liver remodeling. Biochem Biophys Res Commun 272:681-686

Higashiyama R, Inagaki Y, Hong Y et al (2007) Bone marrow-derived cells express matrix metalloproteinases and contribute to regression of liver fibrosis in mice. Hepatology 45:213-222

Horn T, O'Brien T, Schook L, Rutherford M (2000) Acute hepatotoxicant exposure induces TNFR-mediated hepatic injury and cytokine/apoptotic gene expression. Toxicol Sci 54:262-273

Inui T, Nakashima H, Habu Y et al (2005) Neutralization of tumor necrosis factor abrogates hepatic failure induced by alphagalactosylceramide without attenuating its antitumor effect in aged mice. J Hepatol 43:670-678

Khurana S, Mukhopadhyay A (2007) Characterization of the potential subpopulation of bone marrow cells involved in the repair of injured liver tissue. Stem Cells 25:1439-1447

Kitamura K, Nakamoto Y, Akiyama M et al (2002) Pathogenic roles of tumor necrosis factor receptor p55-mediated signals in dimethylnitrosamine-induced murine liver fibrosis. Lab Invest 82:571-583

Liu C, Tao Q, Sun M et al (2010) Kupffer cells are associated with apoptosis, inflammation and fibrotic effects in hepatic fibrosis in rats. Lab Invest 90:1805-1816

Moreira R (2007) Hepatic stellate cells and liver fibrosis. Arch Pathol Lab Med 131:1728-1734

Omori K, Terai S, Ishikawa $\mathrm{T}$ et al (2004) Molecular signature associated with plasticity of bone marrow cell under persistent liver damage by self-organizing-map-based gene expression. FEBS Lett 578:10-20

Parsons CJ, Takashima M, Rippe RA (2007) Molecular mechanisms of hepatic fibrogenesis. J Gastroenterol Hepatol 22(Suppl 1):S79-S84

Pfeffer K, Matsuyama T, Kunding TM et al (1993) Mice deficient for the $55 \mathrm{kd}$ tumor necrosis factor receptor are resistant to endotoxic shock, yet succumb to L. monocytogenes infection. Cell 73:457-467

Robinson S, Scott K, Balkwill F (2002) Chemokine stimulation of monocyte matrix metalloproteinase- 9 requires endogenous TNFalpha. Eur J Immunol 32:404-412

Sakaida I (2008) Autologous bone marrow cell infusion therapy for liver cirrhosis. J Gastroenterol Hepatol 23:1349-1353
Sakaida I, Terai S, Yamamoto N et al (2004) Transplantation of bone marrow cells reduces CCl4-induced liver fibrosis in mice. Hepatology 40:1304-1311

Shibata H, Yoshioka Y, Ohkawa A et al (2008a) The therapeutic effect of TNFR1-selective antagonistic mutant TNF-alpha in murine hepatitis models. Cytokine 44:229-233

Shibata H, Yoshioka Y, Ohkawa A et al (2008b) Creation and X-ray structure analysis of the tumor necrosis factor receptor-1selective mutant of a tumor necrosis factor-alpha antagonist. J Biol Chem 283:998-1007

Simeonova P, Gallucci R, Hulderman T et al (2001) The role of tumor necrosis factor-alpha in liver toxicity, inflammation, and fibrosis induced by carbon tetrachloride. Toxicol Appl Pharmacol 177:112-120

Sudo K, Yamada Y, Moriwaki H, Saito K et al (2005) Lack of tumor necrosis factor receptor type 1 inhibits liver fibrosis induced by carbon tetrachloride in mice. Cytokine 29:236-244

Tacke F, Luedde T, Trautwein C (2009) Inflammatory pathways in liver homeostasis and liver injury. Clin Rev Allergy Immunol 36:4-12

Terai S, Sakaida I (2008) Current status of autologous bone marrow cell infusion therapy for liver cirrhosis patients. Hepatol Res 38: S72-S75

Terai S, Yamamoto N, Omori K et al (2002) A new cell therapy using bone marrow cells to repair damaged liver. J Gastroenterol 37 (Suppl 14):162-163

Terai S, Sakaida I, Yamamoto N et al (2003) An in vivo model for monitoring trans-differentiation of bone marrow cells into functional hepatocytes. J Biochem 134:551-558

Terai S, Sakaida I, Nishina H et al (2005) Lesson from the GFP/CC14 model-translational research project: the development of cell therapy using autologous bone marrow cells in patients with liver cirrhosis. J Hepatobiliary Pancreat Surg 12:203-207

Vaday G, Schor H, Rahat M et al (2001) Transforming growth factorbeta suppresses tumor necrosis factor alpha-induced matrix metalloproteinase-9 expression in monocytes. J Leukoc Biol 69:613-621

Yamada Y, Fausto N (1998) Deficient liver regeneration after carbon tetrachloride injury in mice lacking type 1 but not type 2 tumor necrosis factor receptor. Am J Pathol 152:1577-1589

Yamada Y, Kirillova I, Peschon J et al (1997) Initiation of liver growth by tumor necrosis factor: deficient liver regeneration in mice lacking type I tumor necrosis factor receptor. Proc Natl Acad Sci USA 94:1441-1446 